\title{
ON THE ARENS PRODUCTS AND CERTAIN BANACH ALGEBRAS
}

\author{
BY
}

\section{PAK-KEN WONG}

ABSTRACT. In this paper, we study several problems in Banach algebras concerned with the Arens products.

1. Introduction. Let $A$ be a Banach algebra, $A^{* *}$ its second conjugate space and $\pi_{A}$ the canonical embedding of $A$ into $A^{* *}$. Arens has defined two natural extensions of the product on $A$ to $A^{* *}$. Under either Arens product, $A^{* *}$ becomes a Banach algebra. In $\$ 3$, we show that if $A$ is a semisimple Banach algebra which is a dense two-sided ideal of a semisimple annihilator Banach algebra $B$, then $\pi_{A}(A)$ is a two-sided ideal of $A^{* *}$ (with the Arens product). In particular, a semisimple annihilator Banach algebra has such property. This result greatly generalizes some recent results obtained by the author (see [12, p. $82]$ and $[13$, p. 830$])$.

In $\$ 4$, we study the radical $R^{* *}$ of $A^{* *}$, where $A$ is a semisimple annihilator Banach algebra. We show that, under either Arens product, $R^{* *}$ remains the same and it is the right annihilator of $A^{* *}$. A similar result was obtained by Civin and Yood [5] for the group algebra of a compact abelian group.

$\S 5$ is devoted to the study of semisimple dual Banach algebras which are two-sided ideals of a $B^{*}$-algebra. Let $A$ be a semisimple dual Banach algebra which is a dense subalgebra of a $B^{*}$-algebra $B$ such that $\|\cdot\|$ majorizes $|\cdot|$ on $A$. We show that $A$ is a two-sided ideal of $B$ if and only if, for any orthogonal family of hermitian minimal idempotents $\left\{e_{\lambda}: \lambda \epsilon \Lambda\right\}$ of $B$ and $x \in A, \Sigma_{\lambda} x e_{\lambda}$ and $\Sigma_{\lambda} e_{\lambda} x$ are summable in the norm of $A$. This result was proved by Ogasawara and Yoshinaga [9] for weakly complete commutative dual $A^{*}$-algebras. Finally, by using the above result as well as the result in $\$ 4$, we answer a question of the author affirmatively: if $A$ is a semisimple dual Banach algebra which is a dense two-sided ideal of a $B^{*}$-algebra, then $A$ is Arens regular and $A^{* *} / R^{* *}$ is a semisimple Banach algebra which is a dense two-sided ideal of some $B^{*}$-algebra.

Received by the editors September 5, 1972.

AMS (MOS) subject classifications (1970). Primary 46C05; Secondary 46K99.

Key words and phrases. Arens products, Arens regular, annihilator and dual algebras, $B^{*}$-al gebras.

Copyright $\odot$ 1973, American Mathematical Society 
2. Notation and preliminaries. Definitions not explicitly given are taken from Rickart's book [10].

Let $A$ be a Banach algebra. For each element $a \in A$, let $\operatorname{Sp}_{A}(a)$ denote the spectrum of $a$ in $A$. If $A$ is commutative, $M_{A}$ will denote the carrier space of $A$ and $C_{0}\left(M_{A}\right)$ the algebra of all complex-valued functions on $M_{A}$, which vanishes at infinity. If $A$ is a commutative $B^{*}$-algebra, then $\hat{A}=C_{0}\left(M_{A}\right)$.

Let $A$ be a Banach algebra which is a subalgebra of a Banach algebra $B$. For each subset $E$ of $A, \mathrm{cl}(E)$ (resp. $\mathrm{cl}_{A}(E)$ ) will denote the closure of $E$ in $B$ (resp. $A$ ). We write $\|\cdot\|$ for the norm on $A$ and $|\cdot|$ for the norm on $B$.

For any set $E$ in a Banach algebra $A$, let $l_{A}(E)$ and $r_{A}(E)$ denote the left and right annihilators of $E$ respectively. A Banach algebra $A$ is called an annihilator algebra if $l_{A}(A)=r_{A}(A)=(0)$ and if for every proper closed right ideal $I$ and every proper closed left ideal $J, l_{A}(I) \neq(0)$ and $r_{A}(J) \neq(0)$. If, in addition, $r_{A}\left(l_{A}(I)\right)=I$ and $l_{A}\left(r_{A}(J)\right)=J$, then $A$ is called a dual algebra.

An idempotent $e$ in a Banach algebra $A$ is said to be minimal if $e A e$ is a division algebra. In case $A$ is semisimple, this is equivalent to saying that Ae $(e A)$ is a minimal left (right) ideal of $A$.

In this paper, all algebras and linear spaces under consideration are over the field $C$ of complex numbers.

3. The Arens products and annihilator algebras. Let $A$ be a Banach algebra, $A^{*}$ and $A^{* *}$ the conjugate and second conjugate spaces of $A$, respectively. The two Arens products on $A^{* *}$ are defined in stages according to the following rules (see [1]). Let $x, y \in A, f \in A^{*}, F, G \in A^{* *}$.

(a) Define $f \circ x$ by $(f \circ x)(y)=f(x y)$. Then $f \circ x \in A^{*}$.

(b) Define $G \circ f$ by $(G \circ f)(x)=G(f \circ x)$. Then $G \circ f \in A^{*}$.

(c) Define $F \circ G$ by $(F \circ G)(f)=F(G \circ f)$. Then $F \circ G \in A^{* *}$.

$A^{* *}$ with the Arens product $\circ$ denoted by $\left(A^{* *}, \circ\right)$.

(a') Define $x \circ^{\prime} f$ by $\left(x \circ^{\prime} f\right)(y)=f(y x)$. Then $x \circ^{\prime} f \in A^{*}$.

$\left(b^{\prime}\right)$ Define $f \circ^{\prime} F$ by $\left(f \circ^{\prime} F\right)(x)=F\left(x \circ^{\prime} f\right)$. Then $f \circ^{\prime} F \in A^{*}$.

$\left(c^{\prime}\right)$ Define $F \circ^{\prime} G$ by $\left(F \circ^{\prime} G\right)(f)=G\left(f \circ^{\prime} F\right)$. Then $F \circ G \in A^{* *}$.

$A^{* *}$ with the Arens product $\circ^{\prime}$ denoted by $\left(A^{* *}, \circ^{\prime}\right)$.

Each of these products extends the original multiplication on $A$ when $A$ is canonically embedded in $A^{* *}$. In general, ${ }^{\circ}$ and $\circ^{\prime}$ are distinct on $A^{* *}$. If they coincide on $A^{* *}$, then $A$ is called Arens regular.

Notation. Let $A$ be a Banach algebra. The mapping $\pi_{A}$ will denote the canonical embedding of $A$ into $A^{* *}$.

The left multiplication in $\left(A^{* *}, \circ\right)$ is weakly continuous and the right multiplication in $\left(A^{* *}, \circ^{\prime}\right)$ is weakly continuous (see [1, p. 842]). If $x \in A$ and $F \in$ $A^{* *}$, then $\pi_{A}(x) \circ F=\pi_{A}(x) \circ^{\prime} F$ and $F \circ \pi_{A}(x)=F \circ^{\prime} \pi_{A}(x)$ (see $[1$, p. 843]). 
The following result is useful throughout the paper.

Theorem 3.1. Let $A$ be a semisimple Banach algebra which is a dense twosided ideal of a semisimple annibilator Banacb algebra $B$. Then $\pi_{A}(A)$ is a twosided ideal of $\left(A^{* *}, \circ\right)$. In particular, $\pi_{B}(B)$ is a two-sided ideal of $B^{* *}$ (with the Arens product).

Proof. By [2, p. 3, Proposition 2.2], there exists a constant $k>0$ such that $k\|\cdot\| \geq|\cdot|$ on $A$ and hence by $[2$, p. 3, Theorem 2.3], there exists a constant $M$ such that

$$
\|a b\| \leq M\|a\||b| \text { and }\|b a\| \leq M\|a\||b|
$$

for all $a \in A, b \in B$. Let $e$ be a minimal idempotent of $B$. Since $e A e=e B e=$ $C e$, it follows that $e \in A$. Also if $e$ is a minimal idempotent of $A$, then $e$ is a minimal idempotent of $B$. Therefore $A$ and $B$ have the same minimal idempotents. Let $e$ be a minimal idempotent. Since $A e=B e$, it is easy to see that the norms $\|\cdot\|$ and $|\cdot|$ are equivalent on $A e$. Since $B$ is an annihilator algebra, it follows immediately from $[10$, p. 101, Lemma (2.8.20)] and [10, p. 104, Theorem (2.8.23)] that $B e$ is a reflexive Banach space and hence $A e$ is also reflexive. Let $F \in$ $A^{* *}$. We show that $F \circ \pi_{A}(e) \in \pi_{A}(A)$. Clearly we can assume that $\|F\|=1$. Then by Goldstine's theorem [6, p. 424, Theorem 5] there exists a net $\left\{x_{a}\right\}$ in $A$ such that $\left\|x_{a}\right\| \leq 1$ for all $a$ and $\pi_{A}\left(x_{\alpha}\right) \rightarrow F$ weakly in $A^{* *}$. Hence it follows from the weak continuity of left multiplication that $\pi_{A}\left(x_{a} e\right) \rightarrow F \circ \pi_{A}(e)$ weakly. Since $\left\|x_{\alpha} e\right\| \leq\|e\|$, by [6, p. 425, Theorem 7] we can assume that there exists some $y \in A e$ such that $g\left(x_{a} e\right) \rightarrow g(y)$ for all $g \in(A e)^{*}$. Now for each $f \in A^{*}$, let $f^{\prime}$ be the restriction of $f$ to $A e$. Then we have

$$
\pi_{A}(y)(f)=\lim _{\alpha} f^{\prime}\left(x_{\alpha} e\right)=\lim _{\alpha} \pi_{A}\left(x_{\alpha} e\right)(f)=\left(F \circ \pi_{A}(e)\right)(f)
$$

Therefore, we get

$$
F \circ \pi_{A}(e)=\pi_{A}(y) \in \pi_{A}(A) .
$$

Let $x \in A$. Since the socle $S$ of $B$ is dense in $B$ by $[10, \mathrm{p} .100$, Corollary (2.8.16)], we can write $x=\lim _{n \rightarrow \infty} x_{n}$, where $x_{n} \in S(n=1,2, \ldots)$. Since $S$ is also the socle of $A$, it follows easily from (2) that

$$
F \circ \pi_{A}\left(x_{n}\right) \in \pi_{A}(A) \quad(n=1,2, \ldots) .
$$

Let $f \in A^{* *}$. By (1) we obtain $\left\|a \circ^{\prime} f\right\| \leq M\|f\||a|$ for all $a \in A$ and consequently

$$
\left|\left(F \circ \pi_{A}\left(x_{n}\right)-F \circ \pi(x)\right)(f)\right|=\left|F\left(\left(x_{n}-x\right) \circ f\right)\right| \leq M\|F\|\|f\|\left|x_{n}-x\right| .
$$


Since $x_{n} \rightarrow x$ in $|\cdot|$, we have $F \circ \pi_{A}\left(x_{n}\right) \rightarrow F \circ \pi_{A}(x)$ in $\|\cdot\| \cdot$. Hence it follows from (3) that $F \circ \pi_{A}(x) \in \pi_{A}(A)$. Similarly we can show that $\pi_{A}(x) \circ F \in \pi_{A}(A)$. Therefore $\pi_{A}(A)$ is a two-sided ideal of $\left(A^{* *}, \circ\right)$ and this completes the proof.

Remark. The preceding result generalizes a part of $[13$, p. 830 , Theorem 5.2] as well as [12, p. 82, Theorem 3.3].

Corollary 3.2. Let $A$ be as in Theorem 3.1. Then for every minimal idempotent $e \in A, A^{* *} \circ \pi_{A}(e)$ and $\pi_{A}(e) \circ A^{* *}$ are minimal left and right ideals of $\left(A^{* *}\right.$, o).

Proof. This follows immediately from Theorem 3.1 since $A^{* *} \circ \pi_{A}(e)=\pi_{A}(A e)$ and $\pi_{A}(e) \circ A^{* *}=\pi_{A}(e A)$.

4. The radical of the algebra $\left(A^{* *}, \circ\right)$. This section is devoted to the discussion of the radical of the algebra $\left(A^{* *}, \circ\right)$. The main result in this section is useful in $\$ 5$. Civin and Yood [5] had studied this problem for the group algebra of an infinite locally compact abelian group.

Throughout this section, unless otherwise stated, $A$ will be a semisimple annihilator Banach algebra. Let $R_{1}^{* *}$ (resp. $R_{2}^{* *}$ ) denote the radical of $\left(A^{* *}, \circ\right)$ (resp. $\left(A^{* *}, \circ^{\prime}\right)$ ); $R_{1}^{* *}$ and $R_{2}^{* *}$ may not be zero (see [5, p. 857, Theorem 3.14] and $\left[13\right.$, p. 831, Theorem 5.5]). By Theorem 3.1, $\pi_{A}(A)$ is a two-sided ideal of $\left(A^{* *}\right.$, o).

Theorem 4.1. Let $A$ be a semisimple annibilator Banach algebra. Then the following statements bold:

(i) $R_{1}^{* *}$ is weakly closed.

(ii) $R_{1}^{* *}=\left\{F \in A^{* *}: A^{* *} \circ F=(0)\right\}=\left\{F \in A^{* *}: F \circ^{\prime} A^{* *}=(0)\right\}$.

(iii) $R_{1}^{* *}$ coincides with $R_{2}^{* *}$.

Proof. Let $E_{A}$ be the set of all minimal idempotents of $A$. For each $e \in$ $E_{A}$, let $M=\left(1-\pi_{A}(e)\right) \circ A^{* *}$. We show that $M$ is a maximal modular right ideal of $\left(A^{* *}, \circ\right)$. In fact, suppose there exists a right ideal $M^{\prime}$ of $\left(A^{* *}, \circ\right)$ properly containing $M$. Let $F \in M^{\prime}$ be such that $F \notin M$. Then $\pi_{A}(e) \circ F=F-\left(1-\pi_{A}(e)\right)$ $\circ F \in M^{\prime}$ and $\pi_{A}(e) \circ F \neq 0$. Hence $\left(\pi_{A}(e) \circ A^{* *}\right) \cap M^{\prime} \neq(0)$ and consequently by Corollary $3.2 M^{\prime} \supset \pi_{A}(e) \circ A^{* *}$. Hence $M^{\prime}=A^{* *}$. Therefore $M$ is maximal. Let $\left\{G_{a}\right\}$ be a net in $M$ such that $G_{a} \rightarrow G$ weakly for some $G \in A^{* *}$. Since $\pi_{A}(e) \circ G_{a}=0$ for each $a$, it follows that $\pi_{A}(e) \circ G=0$ and hence $G \in M$. Therefore $M$ is weakly closed. Let

$$
R=\bigcap\left\{\left(1-\pi_{A}(e)\right) \circ A^{* *}: e \in E_{A}\right\} \text { and } T=\left\{F \in A^{* *}: A^{* *} \circ F=(0)\right\} .
$$

Then $R$ is weakly closed and $T \subset R_{1}^{* *} \subset R$. Let $F \in R$. Then $\pi_{A}(e) \circ F=0$ for all $e \in E_{A}$. Since the socle of $A$ is dense in $A$, we have $\pi_{A}(A) \circ F=(0)$. Since 
$\pi_{A}(A)$ is weakly dense in $\left(A^{* *}, \circ\right)$, it follows that $A^{* *} \circ F=(0)$ and so $F \in T$. Consequently $R_{1}^{* *}=R=T$. Similarly by using maximal modular left ideals, we can show that $R_{2}^{* *}=\left\{F \in A^{* *}: F \circ^{\prime} A^{* *}=(0)\right\}$. Let $F \in R_{1}^{* *}, G \in A^{* *}$ and $\left\{x_{a}\right\} \subset A$ such that $\pi_{A}\left(x_{a}\right) \rightarrow G$ weakly. Then $F \circ \pi_{A}\left(x_{a}\right)=F \circ^{\prime} \pi_{A}\left(x_{a}\right) \rightarrow F \circ G$ weakly. Since by Theorem $3.1 F \circ \pi_{A}\left(x_{\alpha}\right) \in R_{1}^{* *} \cap \pi_{A}(A)=(0)$, we have $F \circ^{\prime} G$ $=0$ and so $F \in R_{2}^{* *}$. Hence $R_{1}^{* *} \subset R_{2}^{* *}$. Similarly we can show that $R_{2}^{* *} \subset R_{1}^{* *}$. Therefore they are equal and this completes the proof of the theorem.

Remark 1. Theorem 4.1 (ii) is a generalization of [5, p. 857, Theorem 3.15 (i)].

Remark 2. In general, $R_{1}^{* *} \neq\left\{F \in A^{* *}: F \circ A^{* *}=(0)\right\}$. In fact, let $A$ be the group a lgebra of an infinite compact abelian group. Then by [5, p. 857, Theorem 3.12] $R_{1}^{* *} \neq(0)$. By [5, p. 855, Lemma 3.8], $A^{* *}$ has a right identity. Hence it follows that $\left\{F \in A^{* *}: F \circ A^{* *}=(0)\right\}=(0) \neq R_{1}^{* *}$.

Notation. In the rest of this paper, let $R^{* *}=R_{1}^{* *}=R_{2}^{* *}$.

Corollary 4.2. Suppose $A$ is a semisimple commutative annibilator Banach algebra and $M_{A}$ its carrier space. Let $Q$ be the closed subspace of $A^{*}$ spanned by $M_{A}$ and let $Q^{\perp}=\left\{F \in A^{* *}: F(Q)=(0)\right\}$. Then $Q^{\perp}=R^{* *}$.

Proof. It is well known that $M_{A}$ is discrete. For each $b \in M_{A}$, let $e_{b}$ be the minimal idempotent of $A$ corresponding to the characteristic function of $b$ ([10, p. 168, Theorem (3.6.3)]). For each $b \in M_{A}$ and $x \in A$, we have $x e_{b}=e_{b} x e_{b}$ $=b(x) e_{b}$. Therefore $\left(f \circ e_{b}\right)(x)=f\left(e_{b}\right) b(x)$ for all $f \in A^{*}$. Hence $f \circ e_{b}=f\left(e_{b}\right) b$. Let $F \in A^{* *}$. Then $\left(\pi_{A}\left(e_{b}\right) \circ F\right)(f)=F\left(f \circ e_{b}\right)=f\left(e_{b}\right) F(b)$ for all $f \in A^{*}$. Hence it follows easily that $Q^{\perp}=\left\{F \in A^{* *}: A^{* *} \circ F=(0)\right\}$. Therefore by Theorem 4.1, $Q^{\perp}=R^{* *}$.

Remark. The above result is a generalization of [5, p. 857, Theorem 3.15 (ii)].

Corollary 4.3. Let $M$ be a maximal modular right ideal of $\left(A^{* *}, \circ\right)$. Then either $(l(M))^{2}=(0)$ or there exists a minimal idempotent $e$ of $A$ such that $M=$ $\left(1-\pi_{A}(e)\right) \circ A^{* *}$. In the latter case, $M$ is weakly closed. A similar result bolds for left ideals.

Proof. If $l(M) \subset R^{* *}$, then by Theorem $4.1(l(M))^{2}=(0)$. Suppose $l(M) \not \subset$ $R^{* *}$. We claim that $l(M) \cap \pi_{A}(A) \neq(0)$. Assume this is not so. Then $\pi_{A}(A) \circ$ $l(M) \subset \pi_{A}(A) \cap l(M)=(0)$. Hence $A^{* *} \circ l(M)=(0)$ and so by Theorem 4.1, $l(M)$ $\subset R^{* *}$. This contradiction shows that $l(M) \cap \pi_{A}(A) \neq(0)$. Therefore by $[10, \mathrm{p}$. 98, Lemma (2.8.6)], $l(M) \cap \pi_{A}(A)$ contains a minimal idempotent $\pi_{A}(e)$ of $\pi_{A}(A)$. By the maximality of $M$, we have $M=\left(1-\pi_{A}(e)\right) \circ A^{* *}$. Also $M$ is weakly closed by the proof of Theorem 4.1 and this completes the proof. 
We remark that a similar result for left ideals has been obtained by Civin for the group algebra of an infinite locally compact abelian group (see [3]).

5. Banach algebras which are ideals in a $B^{*}$-algebra. In this section, we study semisimple dual Banach algebras which are two-sided ideals in a $B^{*}$-algebra. There are many examples having such properties in analysis. The algebras $C_{p}$ discussed in [8] and the proper $H^{*}$-algebras are such examples. Unless otherwise stated, $A$ will be a semisimple dual Banach algebra which is a dense subalgebra of a $B^{*}$-algebra $B$ such that $\|\cdot\|$ majorizes $|\cdot|$ on $A$. It is well known that $B$ is also a dual algebra (see $[12$, p. 81]).

The following result is contained in Lemma 5.1 in [7].

Lemma 5.1. $A$ and $B$ bave the same minimal idempotents and the same socle.

Proof. Let $e$ be a minimal idempotent of $A$. Then it is clear that $e$ is a minimal idempotent of $B$. By the proof of [12, p. 82, Lemma 3.2] $\|\cdot\|$ and $|\cdot|$ are equivalent on $A e$ and $B e=A e, e A=e B$. Therefore the socle $S$ of $A$ is a dense two-sided ideal of $B$. Let $f$ be a minimal idempotent of $B$. Then $S f \subset B f \cap S$ and so $B f \subset S \subset A$. Therefore $f$ is a minimal idempotent of $A$. Now it is clear that $S$ is also the socle of $B$.

We shall now give a characterization for $A$ to be a two-sided ideal of $B$.

Theorem 5.2. Let $A$ be a semisimple dual Banach algebra which is a dense subalgebra of a $B^{*}$-algebra $B$ such that $\|\cdot\|$ majorizes $|\cdot|$ on $A$. Then the fol. lowing statements are equivalent:

(i) $A$ is a two-sided ideal of $B$.

(ii) There exists a constant $M>0$ such that $\left\|\sum_{k=1}^{n} e_{k} x\right\| \leq M\|x\|$ and $\left\|\sum_{k=1}^{n} x e_{k}\right\| \leq M\|x\|$, where $x \in A$ and $e_{1}, e_{2}, \cdots, e_{n}$ are any mutually orthogonal bermitian minimal idempotents of $B$.

(iii) For any orthogonal family of hermitian minimal idempotents $\left\{e_{\lambda}: \lambda \in \Lambda\right\}$ of $B$ and $x \in A, \Sigma_{\lambda} x e_{\lambda}$ and $\Sigma_{\lambda} e_{\lambda} x$ are summable in the norm of $A$ and especially when $\left\{e_{\lambda}: \lambda \in \Lambda\right\}$ is a maximal family, $x=\Sigma_{\boldsymbol{\lambda}} x e_{\boldsymbol{\lambda}}=\Sigma_{\boldsymbol{\lambda}} e_{\boldsymbol{\lambda}} x$ in $A$.

Proof. We know that $B$ is a dual algebra and $A$ and $B$ have the same minimal idempotents and the same socle by Lemma 5.1.

(i) $\Rightarrow$ (ii). Suppose (i) holds. Then by [2, p. 3, Theorem 2.3] there exists a constant $M$ such that $\left\|\sum_{k=1}^{n} e_{k} x\right\| \leq M\left|\Sigma_{k=1}^{n} e_{k}\right|\|x\|=M\|x\|$. Similarly, $\left\|\sum_{k=1}^{n} x e_{k}\right\| \leq M\|x\|$ and this proves (ii).

(ii) $\Rightarrow$ (iii). Suppose (ii) holds. Let $\left\{e_{\lambda}: \lambda \in \Lambda\right\}$ be an orthogonal family of hermitian minimal idempotents of $B$ and $x \in A$. Let $\left\{E_{\gamma}: \gamma \in \Gamma\right\}$ be the direct set of all finite sums $e_{\lambda_{1}}+e_{\lambda_{2}}+\cdots+e_{\lambda_{n}}\left(\lambda_{k} \in \Lambda\right.$ and $\left.n=1,2, \cdots\right)$. Since $\left\|x E_{\gamma}\right\|<M\|x\|$ by (ii), it follows from the Alaoglu theorem that $\left\{\pi_{A}\left(x E_{\gamma}\right)\right\}$ has 
weak limit points in $A^{* *}$. Let $F \in A^{* *}$ be a weak limit point of $\left\{\pi_{A}\left(x E_{\gamma}\right)\right\}$. Then for any $y \in A, \pi_{A}(y) \circ F$ is a weak limit point of $\pi_{A}\left(y x E_{\gamma}\right)$. Since $A$ is a dual algebra, by Theorem $3.1 \pi_{A}(y) \circ F \in \pi_{A}(A)$. Let $\left\{e_{a}: a \in \Delta\right\}$ be a maximal orthogonal family of hermitian minimal idempotents of $B$ containing $\left\{e_{\lambda}: \lambda \in \Lambda\right\}$. Then it is easy to see that $\pi_{A}(y) \circ F \circ \pi_{A}\left(e_{\alpha}\right)=\pi_{A}\left(y x e_{\alpha}\right)(\alpha \in \Delta)$. Since $\left\{e_{a}\right.$ : $a \in \Lambda\}$ is maximal, it follows that $\pi_{A}(y) \circ F=\pi_{A}(y x)$ (see [9, p. 21]). Hence $\left\{y x E_{\gamma}\right\}$ converges weakly to $y x$ and so by the Orlicz-Banach theorem [6, p. 93], $\Sigma_{\boldsymbol{\lambda}} y x e_{\boldsymbol{\lambda}}$ is summable in the norm of $A$. Since $A$ is a dual algebra by $[10, \mathrm{p} .91$, Corollary (2.8.3)] $x \in \mathrm{cl}_{A}(A x)$. Hence, for any given $\epsilon>0$, there exists some $z \in A$ such that $\|x-z x\|<\epsilon$. Now by (ii) we have $\left\|x E_{\gamma}\right\| \leq M\|x-z x\|+\left\|z x E_{\gamma}\right\|$ $<M \epsilon+\left\|z x E_{\gamma}\right\|$. Since $\Sigma_{\lambda} z x e_{\lambda}$ is summable in $\|\cdot\|$ and $\epsilon$ is arbitrary, it follows that $\Sigma_{\boldsymbol{\lambda}} x e_{\boldsymbol{\lambda}}$ is summable in $\|\cdot\|$. If $\left\{e_{\boldsymbol{\lambda}}: \lambda \in \Lambda\right\}$ is a maximal family, then it is easy to see that $x=\Sigma_{\lambda} x e_{\lambda}$. Similarly we can show that $x=\Sigma_{\lambda} e_{\lambda} x$ and this proves (iii)

(iii) $\Rightarrow$ (i). Suppose (iii) holds. Let $x \in A$ and $y \in B$. We shall show that $x y \in B$. Since any element of $B$ is a linear combination of positive elements, we may assume that $y$ is a positive element. We also assume that $x \neq 0$ and $y \neq 0$. Let $E$ be a maximal commutative *-subalgebra of $B$ containing $y$. Then the carrier space $M_{E}$ of $E$ is discrete. For each $\lambda \in M_{E}$, let $e_{\lambda}$ be the element of $E$ corresponding to the characteristic function of $\lambda$. Then $\left\{e_{\lambda}: \lambda \in M_{E}\right\}$ is a maximal orthogonal family of hermitian minimal idempotents in $B$. Since $y \in E$ and $\operatorname{SP}_{E}(y)>0$, we have $y e_{\lambda}=\beta_{\lambda} e_{\lambda}$, where $\beta_{\lambda} \geq 0$ for all $\lambda$ and $\beta_{\lambda} \leq|y|$. Since $B$ is a dual $B^{*}$-algebra, by the proof of (ii) $\Rightarrow$ (iii) (or $[9$, p. 22, Corollary 1]) $x y=\Sigma_{\lambda} x y e_{\lambda}$ in $|\cdot|$ and so there exists only a countable number of $e_{\lambda}$ for which $x y e_{\lambda} \neq 0$, say $e_{1}, e_{2}, \ldots$. For any two positive integers $m, n(m<n)$, let $z_{m}^{n}=\sum_{k=m}^{n} x y e_{k}=\sum_{k=m}^{n} \beta_{k} x e_{k}$. Then $z_{m}^{n} \in A$. We shall show that $\left\{\sum_{k=1}^{n} x y e_{k}\right\}$ is Cauchy sequence in $A$. Clearly, we can assume that each $z_{m}^{n}$ is a nonzero element. Choose $f \in A^{*}$ such that $\|f\|=1$ and $f\left(z_{m}^{n}\right)=\left\|z_{m}^{n}\right\|$ by the Hahn-Banach theorem. Then $f\left(z_{m}^{n}\right)=\sum_{k=m}^{n} \beta_{k} f\left(x e_{k}\right)$. Write $f\left(x e_{k}\right)=a_{k}+i b_{k}$, where $a_{k}, b_{k}$ are real numbers. Then we have

$$
\sum_{k=m}^{n} \beta_{k} f\left(x e_{k}\right)=\sum_{k=1}^{n} \beta_{k} a_{k}=\left\|z_{m}^{n}\right\|>0 \text {. }
$$

Since $\beta_{k} \geq 0$, there exists some $a_{k}>0$. Let $\left\{a_{k^{\prime}}\right\} \subset\left\{a_{k}\right\}_{k=m}^{n}$ such that $a_{k^{\prime}}>0$. Then we have

$$
\begin{aligned}
& \left\|\sum_{k=m}^{n} x y e_{k}\right\|=\left\|z_{m}^{n}\right\|=\sum_{k=m}^{n} \beta_{k} a_{k} \leq \sum_{k^{\prime}} \beta_{k^{\prime}} a_{k^{\prime}} \\
& \leq|y| \sum_{k^{\prime}} a_{k^{\prime}} \leq|y|\left|\sum_{k^{\prime}} f\left(x e_{k^{\prime}}\right)\right| \leq|y|\|f\|\left\|\sum_{k^{\prime}} x e_{k^{\prime}}\right\|=|y|\left\|\sum_{k^{\prime}} x e_{k^{\prime}}\right\| .
\end{aligned}
$$


Hence it follows from the assumption that $\left\{\sum_{i=1}^{n} x y e_{k}\right\}$ is a Cauchy sequence in $A$. Therefore, there exists an element $z \in A$ such that $z=\sum_{k=1}^{\infty} x y e_{k}$ in $\|\cdot\|$. Also $x y=\sum_{k=1}^{\infty} x y e_{k}$ in $1 \cdot 1$. Hence it follows that $x y=z \in A$. Similarly we can show that $y x \in A$. Thus $A$ is a two-sided ideal of $B$ and this completes the proof of the theorem.

Remark 1. (i) $\Rightarrow$ (iii) in the above theorem was obtained by Ogasawara and Yoshinaga for $A^{*}$-algebras (see [9, p. 30, Theorem 16]). Also (iii) $\Leftrightarrow$ (i) was proved by them for weakly complete commutative $A^{*}$-algebras (see $[9$, p. 35, Theorem 2.3]). Some arguments in the proof of (ii) $\Rightarrow$ (iii) of Theorem 5.2 are similar to those in the proof of [9, p. 30, Theorem 16].

Remark 2. If $B$ is not a $B^{*}$-algebra, then Theorem 5.2 is not true. In fact, let $G$ be an infinite compact group and let $A$ be the algebra of all continuous functions on $G$, normed by the maximum of the absolute value. It is well known that $L_{2}(G)$ is an $A^{*}$-algebra and $A$ is a dual $A^{*}$-algebra which is a dense twosided ideal of $L_{2}(G)$. However condition (iii) of Theorem 5.2 is not valid for $A$. Since $L_{2}(G)$ is a proper $H^{*}$-algebra, condition (iii) holds for $L_{2}(G)$.

Corollary 5.3. Let $A$ be a reflexive $A^{*}$-algebra which is a dense subalge. bra of a $B^{*}$-algebra $B$. Then the following statements are equivalent:

(i) $A$ is a two-sided ideal of $B$.

(ii) $A$ is a dual algebra and, for any orthogonal family of bermitian minimal idempotents $\left\{e_{\lambda}: \lambda \in \Lambda\right\}$ of $B$ and $x \in A$, the set $\left\{\sum_{k=1}^{n} e_{\lambda_{k}} x: \lambda_{k} \in \Lambda\right\}$ is bounded in $A$.

Proof. (i) $\Rightarrow$ (ii). This follows immediately from [13, p. 831, Theorem 5.4] and Theorem 5.2 (ii).

(ii) $\Rightarrow$ (i). Suppose (ii) holds. Since $A$ is reflexive, $\left\{\sum_{k=1}^{n} e_{\lambda_{k}}^{x:} \lambda_{k} \in \Lambda\right\}$ has weak limit points in $A$. By the proof of Theorem 5.2, it has a unique weak limit point and so $\Sigma_{\lambda} e_{\lambda} x$ is summable in the norm of $A$. Therefore $A$ is a two-sided ideal of $B$ by Theorem 5.2.

It is well known that a reflexive $B^{*}$-algebra is finite dimensional. The following corollary is a generalization of this result.

Corollary 5.4. Let $A$ be a reflexive $A^{*}$-algebra which is a dense two-sided ideal of a $B^{*}$-algebra $B$. If $A$ bas an approximate identity, then $A$ is finite dimensional.

Proof. It follows immediately from [5, p. 855, Lemma 3.8] and Corollary 5.3 that $A$ is a dual algebra with an identity. Therefore $A$ is finite dimensional.

It is well known that $B$ is Arens regular if $B$ is a $B^{*}$-algebra. Let $A$ be a semisimple dual Banach algebra which is a dense two-sided ideal of a $B^{*}$-algebra $B$. Is $A$ Arens regular? This question was asked in $[13$, p. 833]. We shall answer this question affirmatively. 
Notation. In the rest of this section, $B^{* *}$ with the Arens product will be denoted by $\left(B^{* *}, *\right)$.

Lemma 5.5. Suppose $B$ is a dual $B^{*}$-algebra and $S$ its socle. Let $B^{\prime}$ be the closed subspace of $B^{*}$ spanned by $\pi_{B}(x) * g$, where $x \in S$ and $g \in B^{*}$. Then $B^{*}$ coincides with $B^{\prime}$.

Proof. Suppose this is not true. Then there exists a nonzero linear functional $F \in B^{* *}$ such that $F\left(B^{\prime}\right)=(0)$. Hence, for all $x \in S,\left(F * \pi_{B}(x)\right)(g)=$ $F\left(\pi_{B}(x) * g\right)=0$. Since $S$ is weakly dense in $B^{* *}$, it follows that $F * B^{* *}=(0)$. Since $B^{* *}$ is a $B^{*}$-algebra, we have $F=0$, a contradiction. Therefore $B^{*}$ coincides with $B^{\prime}$.

In the rest of this section, let $A$ be a semisimple Banach algebra which is a dense two-sided ideal of a $B^{*}$-algebra $B$. By [2, p. 3, Proposition 2.2], there exists a constant $k$ such that $k\|\cdot\| \geq|\cdot|$ on $A$ and consequently by [2, p. 3, Theorem 2.3] there exists a constant $M$ such that $\|a b\| \leq M\|a\||b|$ and $\|b a\| \leq$ $M\|a\||b|$ for all $a \in A, b \in B$. For each $g \in B^{*}$, let $g_{A}$ denote the restriction of $g$ to $A$. Then it is easy to see that $g_{A} \in A^{*}$. For every element $F \in A^{* *}$, let $\widetilde{F}$ be the linear functional on $B^{*}$ defined by $\widetilde{F}(g)=F\left(g_{A}\right)\left(g \in B^{*}\right)$. Then $\widetilde{F} \in B^{* *}$. Let $b \in B$ and $f \in A^{*}$. Define $(f \circ b)(a)=f(b a)(a \in A)$. Since $|(f \circ b)(a)| \leq M\|f\||b|\|a\|$, it follows that $f \circ b \in A^{*}$.

As before, let $R^{* *}$ denote the radical of $\left(A^{* *}, \circ\right)$.

Lemma 5.6. Suppose $A$ is an annibilator algebra. Then the following statements bold:

(i) For each $R \in R^{* *}$ and $g \in B^{*}$, we have $\tilde{R}(g)=0$.

(ii) $R^{* *}$ is the left and right annibilator of $\left(A^{* *}, 0\right)$.

Proof. (i) Let $g \in B^{*}$. By Lemma 5.5, we can write $g=\lim _{n} g_{n}$ where $g_{n}=\sum_{i=1}^{m} \pi_{B}\left(x_{i}^{n}\right) * g_{i}^{n}$ with $x_{i}^{n} \in S$ (the socle of $B$ ) and $g_{i}^{n} \in B^{*}$. Clearly $x_{i}^{n} \in$ $A$. Then for each $R \in R^{* *}$, we have

$$
\widetilde{R}(g)=\lim _{n} \sum_{i=1}^{m} \tilde{R}\left(\pi_{B}\left(x_{i}^{n}\right) * g_{i}^{n}\right)=\lim _{n} \sum_{i=1}^{m}\left(R \circ \pi_{A}\left(x_{i}^{n}\right)\right)\left(\left(g_{i}^{n}\right)_{A}\right) .
$$

By Theorem 4.1, we have $R \circ \pi_{A}\left(x_{k}^{n}\right)=0$ and therefore $\tilde{R}(g)=0$. This proves (i).

(ii) For each $F \in A^{* *}$ and $f \in A^{*}$, define $\tilde{f}_{F}(b)=F(f \circ b)(b \in B)$. Then it is easy to see that $\tilde{f}_{F} \in B^{*}$ and $\left(\tilde{f}_{F}\right)_{A}=F \circ f$. Then for all $R \in R^{* *}$, we have $(R \circ F)(f)=R(F \circ f)=\widetilde{R}\left(\widetilde{f}_{F}\right)$. Therefore by $(\mathrm{i}), R \circ F=0$ and so $R^{* *} \circ A^{* *}=(0)$.

By Theorem 4.1, we also have $A^{* *} \circ R^{* *}=(0)$ and this completes the proof.

Now we are ready to prove the following result: 
Theorem 5.7. Let $A$ be a semisimple dual Banach algebra which is a dense two-sided ideal of a $B^{*}$-algebra. Then the following statements bold:

(i) $A$ is Arens regular.

(ii) $A^{* *} / R^{* *}$ is a semisimple Banach algebra which is a dense two-sided ideal of some $B^{*}$-algebra.

Proof. (i) Let $\left\{e_{\lambda}: \lambda \in \Lambda\right\}$ be a maximal orthogonal family of hermitian minimal idempotents in $B$. Let $\left\{E_{\beta}\right\}$ be the direct set of all finite sums $e_{\lambda_{1}}+$ $e_{\lambda_{2}}+\cdots+e_{\lambda_{n}}\left(\lambda_{n} \in \Lambda, n=1,2, \cdots\right)$. Let $F$ and $G$ be two functionals in $A^{* *}$. Since $\left\|\stackrel{n}{F} \circ \pi_{A}\left(E_{\beta}\right)\right\| \leq M\|F\|\left|E_{\beta}\right|=M\|F\|$, it follows from Alaoglu's theorem that $\left\{F \circ \pi_{A}\left(E_{\beta}\right)\right\}$ has weak limit points in $A^{* *}$. Let $\left\{E_{\alpha}\right\}$ be a subnet of $\left\{E_{\beta}\right\}$ and $F_{1} \in A^{* *}$ such that $F \circ \pi_{A}\left(E_{\alpha}\right) \rightarrow F_{1}$ weakly. By a similar argument, there exists a subnet $\left\{E_{\gamma}\right\}$ of $\left\{E_{a}\right\}$ and $G_{1} \in A^{* *}$ such that $\pi_{A}\left(E_{\gamma}\right) \circ G \rightarrow G_{1}$ weakly. Let $a \in A$. Then by Theorem 5.2, $a=\Sigma_{\lambda} e_{\lambda} a$ in $\|\cdot\|$. Hence $E_{\beta} a \rightarrow a$ weakly. Thus $E_{\gamma} a \rightarrow a$ weakly. Since $F \circ \pi_{A}(x)=F \circ \pi_{A}(x)$ for all $x \in A$, we have $F_{1} \circ \pi_{A}(a)=$ weak limit $F \circ \pi_{A}\left(E_{\gamma} a\right)=F \circ \pi_{A}(a)$. Since $\pi_{A}(A)$ is weakly dense in $A^{* *}$, it follows that $\left(F-F_{1}\right) \circ^{\prime} A^{* *}=(0)$ and so by Theorem 4.1, $F-F_{1} \epsilon$ $R^{* *}$. Similarly we can show that $G_{1}-G \in R^{* *}$. Then by Lemma 5.6, we have

$$
\begin{aligned}
F \circ G & =\left(F_{1}+\left(F-F_{1}\right)\right) \circ G=F_{1} \circ G \\
& =\underset{\gamma}{\operatorname{weak}} \lim F \circ \pi_{A}\left(E_{\gamma}\right) \circ G=\underset{\gamma}{\operatorname{wak}} \lim F \circ\left(\pi_{A}\left(E_{\gamma}\right) \circ G\right) \\
& =F \circ^{\prime} G_{1}=F \circ^{\prime} G .
\end{aligned}
$$

Therefore $A$ is Arens regular by definition and this proves (i).

(ii) Now the algebra $A^{* *} / R^{* *}$ is a semisimple Banach algebra. For each $a \in A$ and $f \in A^{*}$, define $(f * a)(b)=f(a b)(b \in B)$. Then $f * a \in B^{*}$. For each $F \in A^{* *}$, we write $\dot{F}=F+R^{* *}$ and define a mapping $\Phi$ from $A^{* *} / R^{* *}$ into $B^{* *}$ by $\Phi(\dot{F})=\widetilde{F}\left(F \in A^{* *}\right)$. Suppose $\dot{\Phi}(\dot{F})=0$. Then $\widetilde{F}(f * a)=0$ and therefore $\left(\pi_{A}(a) \circ F\right)(f)=0$ for all $a \in A$ and $f \in A^{*}$. Consequently $F \in R^{* *}$ and therefore $\dot{F}=R^{* *}$. Hence it follows that $\Phi$ is an isomorphism of $A^{* *} / R^{* *}$ into $B^{* *}$. For each $g \in B^{*}$, we have $\left\|g_{A}\right\| \leq k|g|$. Since by Lemma 5.5 (i), $R\left(g_{A}\right)$ $=0$ for all $R \in R^{* *}$, straightforward calculations yield that $k\|F+R\| \geq|\widetilde{F}|$ for all $F \in A^{* *}$. Hence $k\|\dot{F}\| \geq|\widetilde{F}|$ and consequently $\Phi$ is continuous. For each $H \in B^{* *}$, define $(H \circ f)(a)=H(f * a)\left(f \in A^{*}, a \in A\right)$. Then $H \circ f \in A^{*}$. For each $F \in A^{* *}$, define $F_{H}(f)=F((H \circ f))\left(f \in A^{*}, F \in A^{* *}\right)$. Then $F_{H} \in A^{* *}$. For each $g \in B^{*}$, we have

$$
\widetilde{F}_{H}(g)=F\left(\left(H \circ g_{A}\right)\right)=F\left((H * g)_{A}\right)=(\widetilde{F} * H)(g) .
$$

Therefore $\widetilde{F} * H=\widetilde{F}_{H}$. Consequently $\Phi\left(A^{* *} / R^{* *}\right)$ is a two-sided ideal of $B^{* *}$. Let $Q$ be the norm closure of $\Phi\left(A^{* *} / R^{* *}\right)$ in $B^{* *}$. Then $Q$ is a closed two- 
sided ideal of $B^{* *}$. Since $B^{* *}$ is a $B^{*}$-algebra, so is $Q$. This completes the proof of the the orem.

Remark. We know that the above result is not true for arbitrary dual $A^{*}$-algebras (see [13, p. 833, Remark]). Also if $A$ is a dual $A^{*}$-algebra which is Arens regular, $A$ may not be a two-sided ideal of its completion in an auxiliary norm; in fact, $A$ can be reflexive (see [9, p. 35]).

Let $\mathbb{Q}=A^{* *} / R^{* *}$. Clearly, we can identify $A$ as a closed two-sided ideal of $\mathbb{Q}$.

Corollary 5.8. Let $A$ be as in Theorem 5.7. Then $\mathbb{Q}$ coincides with $A$ if and only if the socle of $\mathbb{A}$ is dense in $\mathbb{Q}$.

Proof. We use the notation in the proof of Theorem 5.7. Suppose the socle of $\mathbb{Q}$ is dense in $\mathbb{A}$. Then $Q$ is a dual $B^{*}$-algebra. For each minimal idempotent $e \in Q$ and $b \in B$, we have $e=k e \pi_{B}(b) e \in \pi_{B}(B)$, where $k$ is a constant. Hence it follows that $Q=B$. Now it is easy to see that $\mathbb{Q}^{2} \subset A$. Since the socle of $\mathbb{Q}$ is dense in $\mathbb{Q}, \mathbb{Q} \subset A$ and so $\mathbb{Q}=A$. The converse of the corollary is clear and this completes the proof.

If $A$ is reflexive, then it is clear that $A^{* *}$ is semisimple. However, in general, $A^{* *}$ may not be semisimple as shown in $[13$, p. 831 , Theorem 5.5].

Corollary 5.9. Let $A$ be as in Theorem 5.7. Then $A^{* *}$ is semisimple if and only if $A^{*}$ is spanned by $\pi_{A}(x) \circ f$, where $f \in A^{*}$ and $x \in A$.

Proof. Suppose $A^{*}$ is spanned by $\pi_{A}(x) \circ f$. Let $F \in R^{* *}$. Since $F \circ \pi_{A}(x)$ $=0$ for all $x \in A$, it follows that $F(f)=0$ for all $f \in A^{*}$. Hence $F=0$. The converse of the corollary follows immediately from the proof of Lemma 5.5.

Let $A$ be a Banach *algebra. For all $x \in A, f \in A^{*}$ and $F \in A^{* *}$, we define

$$
f^{*}(x)=\overline{f\left(x^{*}\right)} \text { and } F^{*}(f)=\overline{F\left(f^{*}\right)}
$$

where the bar denotes the complex conjugation. If $A$ is a $B^{*}$-algebra, then $A^{* *}$ is a $B^{*}$-algebra under the involution $F \rightarrow F^{*}$ (see $[11$, p. 192]).

Corollary 5.10. Let $A$ be a dual $A^{*}$-algebra which is a dense two-sided ideal of a $B^{*}$-algebra $B$. Then $\left(A^{* *}\right.$, o) is a *algebra and $A^{* *} / R^{* *}$ is an $A^{*}$. algebra which is a dense two-sided ideal of a $B^{*}$-algebra.

Proof. By Theorem 5.7, $A$ is Arens regular and so $A^{* *}$ is a *-algebra under the involution $F \rightarrow F^{*}$ by $\left[11\right.$, p. 186 , Theorem 1]. Clearly $R^{* *}$ is a *-ideal of $A^{* *}$. Now the corollary follows easily from Theorem 5.7.

It was asked in $[13$, p. 833$]$ whether the algebra $C_{p}^{* *}$ is semisimple. If 
$1<p<\infty$, then $C_{p}$ is reflexive (see [8, p. 265]) and, therefore, it is semisimple. If $p=1$, then by [12, p. 831, Theorem 5.5], $C_{1}^{* *}$ is not semisimple unless it is finite dimensional.

\section{REFERENCES}

1. R. Arens, The adjoint of a bilinear operation, Proc. Amer. Math. Soc. 2 (1951), 839-848. MR 13, 659.

2. B. A. Barnes, Banach algebras which are ideals in a Banach algebra, Pacific J. Math. 38 (1971), 1-7.

3. P. Civin, Annihilators in the second conjugate algebra of a group algebra, Pacific J. Math. 12 (1962), 855-862. MR $26 \# 2894$.

4. - Ideals in the second conjugate algebra of a group algebra, Math. Scand. 11 (1962), 161-174. MR 27 \#5139.

5. P. Civin and B. Yood, The second conjugate space of a Banach algebra as an algebra, Pacific J. Math. 11 (1961), 847-870. MR 26 \#622.

6. N. Dunford and J. T. Schwartz, Linear operators. I: General theory, Pure and Appl. Math., vol. 7, Interscience, New York, 1958. MR 22 \#302.

7. T. Husain and P. K. Wong, Quasi-complemented algebras, Trans. Amer. Math. Soc. 174 (1972), 141-154.

8. C. A. McCarthy, $c_{p}$, Israel J. Math. 5 (1967), 249-271. MR 37 \#35.

9. T. Ogasawara and K. Yoshinaga, Weakly completely continuous Banach *-algebras, J. Sci. Hiroshima Univ. Ser. A 18 (1954), 15-36. MR 16, 1126.

10. C. E. Rickart, General theory of Banach algebras, University Series in Higher Math., Van Nostrand, Princeton, N. J., 1960. MR 22 \#5903.

11. M. Tomita, The second dual of a $C^{*}$-algebra, Mem. Fac. Kyushu Univ. Ser. A. 21 (1967), 185-193. MR $36 \# 6955$.

12. P. K. Wong, On the Arens product and annihilator algebras, Proc. Amer. Math. Soc. 30 (1971), 79-83. MR 43 \#6724.

13. - Modular annihilator $A^{*}$-algebras, Pacific J. Math. 37 (1971), 825-834.

14. - The Arens product and duality in $B^{*}$-algebras. II, Proc Amer. Math. Soc. 27 (1971), 535-538. MR 43 \#933.

15. - On the Arens product and commutative Banach algebras, Proc. Amer. Math. Soc. 37 (1972), 111-113.

DEPARTMENT OF MATHEMATICS, SETON HALL UNIVERSITY, SOUTH ORANGE, NEW JERSEY 07079 